\title{
Correlation of xeroderma pigmentosum complementation group $F$ expression with gastric cancer and prognosis
}

\author{
PEILIN $\mathrm{LI}^{1}$ and YUANZHONG MA ${ }^{2}$ \\ ${ }^{1}$ Department of General Surgery, The Second People's Hospital of Dezhou City, Dezhou, Shandong 253000; \\ ${ }^{2}$ Department of Anesthesia, Dezhou People's Hospital, Dezhou, Shandong 253014, P.R. China
}

Received December 28, 2017; Accepted July 9, 2018

DOI: $10.3892 / \mathrm{ol} .2018 .9529$

\begin{abstract}
Correlation of xeroderma pigmentosum complementation group F (XPF) expression with gastric cancer and prognosis was investigated. We randomly selected 76 gastric cancer patients who were admitted to the Second People's Hospital of Dezhou City and received treatment, and detected XPF expression in gastric cancer tissues (observation group) and normal gastric mucosa adjacent to tumor (control group) via immunohistochemistry. Correlation between XPF expression and clinicopathological indicators of gastric cancer was verified via single-factor Chi-square test. Cox's proportional hazard regression model was used in the analysis of influencing factors of patient's prognosis, and Kaplan-Meier was used to analyze the survival rates of XPF-positive and -negative patients. In the observation group, the XPF-positive rate was significantly higher than that in the control group with a statistically significant difference $(\mathrm{P}<0.05)$. Single-factor analysis showed that XPF expression was correlated with the family history and Laurén classification $(\mathrm{P}<0.05)$. Kaplan-Meier survival analysis revealed that the survival time of XPF-positive patients was shorter than that of XPF-negative patients $(\mathrm{P}<0.05)$. Multifactorial analysis using Cox's hazards model suggested that XPF was an independent factor affecting the prognosis of gastric cancer $(\mathrm{P}<0.05)$. In conclusion, XPF expression plays an important role in the occurrence and development of gastric cancer, and a high expression of XPF suggests a poor prognosis of gastric cancer patients.
\end{abstract}

\section{Introduction}

Gastric cancer is the most common gastrointestinal tumor in the world. Variations in the dietary structure have led to

Correspondence to: Dr Yuanzhong Ma, Department of Anesthesia, Dezhou People's Hospital, 1751 Xinhu Avenue, Dezhou, Shandong 253014, P.R. China

E-mail: dezh1133@163.com

Key words: xeroderma pigmentosum complementation group $\mathrm{F}$, gastric cancer, immunohistochemistry a gradual increase in the incidence of gastric cancer worldwide. Both the incidence and mortality rates of gastric cancer rank behind lung cancer, and are ranked second in malignant tumors (1). Since most patients have a relatively poor awareness of health care, detection and diagnosis of gastric cancer occur once the disease has already progressed into the middle or advanced stage (2).

The causes and pathogenesis of gastric cancer are very complex, and the occurrence is induced by multiple factors and procedures as well as progressive development. For example, helicobacter pylori infection, environment, diet and genetic factors are of great significance for the occurrence of gastric cancer (3). Affected by various factors, carcinogens lead to damage to DNA in human cells and genetic damage will continuously accumulate without any timely repair, eventually inducing abnormal proliferation and apoptosis of cells, thus leading to gastric cancer (4). Nucleotide excision repair (NER) system is the main and most important damage-repair pathway of DNA in the human body. As a kind of NER gene, xeroderma pigmentosum complementation group F (XPF) can exert a rate-limiting effect in the repair pathway, which leads to carcinogenesis and resistance to chemotherapy $(5,6)$. In particular, a high expression of XPF increases the risk of skin cancer (7).

In this study, we investigated the XPF expression of gastric cancer patients, and analyzed the correlation of XPF expression with occurrence and prognosis of gastric cancer.

\section{Patients and methods}

General material. We randomly selected a total of 76 patients who were admitted to the Second People's Hospital of Dezhou City (Dezhou, China) for treatment. Inclusion criteria for the study were: i) patients whose diagnosis of progressive gastric cancer was confirmed by pathological biopsy using electronic gastroscope, contrast-enhanced computed tomography (CT) or magnetic resonance imaging (MRI); ii) patients who received surgical treatment and whose excised lesions were taken as specimens; iii) patients who signed the informed consent. Exclusion criteria were: i) patients whose maximal diameter of tumor was $>10 \mathrm{~cm}$; ii) patients with other malignant tumors. Normal gastric mucosa tissues adjacent to the lesion of gastric cancer were taken as the control group. The basic characteristics of the patients are shown in Table I. 
The study was approved by the Ethics Committee of the Second People's Hospital of Dezhou City. Patients signed the informed consent.

\section{Methods}

Collection of specimens. Lesion tissues that were excised in the surgical treatment of patients and part of the normal gastric mucosa tissue adjacent to the tumor of gastric cancer were taken as specimens. Once obtained, fresh specimens were regularly dehydrated in an ascending series of alcohol rinses, treated using dimethylbenzene for transparency, and embedded in paraffin.

Experimental equipments and material. Slicing machine (Leica, Mannheim, Germany), micropipette (Eppendorf, Hamburg, Germany), microscope (BX40, Olympus Corporation; Tokyo, Japan) electrothermostat, centrifuge tubes in different specifications and autoclave were used. Experimental reagents included: i) primary rabbit anti-human polyclonal antibody; ii) secondary goat anti-rabbit polyclonal antibody; iii) DAB (3,3'-diaminobenzidine) color development kit (Fuzhou Maixin Biotechnology Co., Ltd.); iv) other assistant reagents: dimethylbenzene, buffer, anhydrous ethanol, neutral balsam and hydrogen peroxide solution.

Immunohistochemistry. Experimental procedures were: i) Histological section: Serial sectioning was performed using the slicing machine to cut the paraffin-embedded tissues into 6-8 sections with a thickness of 4-5 $\mu \mathrm{m}$; ii) Dewaxing: After sections were heated in an electrothermostat for $45 \mathrm{~min}$ at $70^{\circ} \mathrm{C}, 5 \mathrm{ml}$ of dimethylbenzene was added onto the sections twice ( 5 min each time) followed by swing in slow motion; then the sections were rinsed by cool anhydrous ethanol twice (5 min each time); when there was no up-floated floccules in the solution, ethanol in different concentrations $(95,85$ and $75 \%$ ) was sequentially added into the solution followed by 5 min of standing, respectively; and slices were then rinsed using distilled water; iii) Phosphate-buffered saline (PBS) rinsing and incubation: Sections were taken out and placed in an incubator, in which $5 \mathrm{ml}$ of PBS was added for rinsing 3 times. After the PBS was fully removed, $50 \mu 1$ hydrogen dioxide solution ( $3 \%$ ) was added onto each section followed by incubation for $10 \mathrm{~min}$ at $20^{\circ} \mathrm{C}$ in an electrothermostat to block the activity of endogenous peroxidase. The sections were then washed using $5 \mathrm{ml}$ PBS (3 min each time), after which the PBS was removed, $50 \mu 1$ primary rabbit anti-human polyclonal antibody (1:300; cat. no. 13465; Cell Signaling Technology, Inc.; Danvers, MA, USA) was added onto each section that was later incubated overnight at $4^{\circ} \mathrm{C}$ in a refrigerator, and the next day, sections were rinsed using PBS 3 times. Secondary goat anti-rabbit polyclonal antibody (1:1,000; cat. no. 7074; Cell Signaling Technology, Inc.) was also added onto each section for $15 \mathrm{~min}$ of incubation at $20^{\circ} \mathrm{C}$ in an incubator, and the sections were washed using PBS three times. For color development: DAB kit, and reagents A, B and C (each $50 \mu \mathrm{l}$ ) were used to prepare the DAB color development solution. After the extra water on each section was cleaned, $50 \mu 1$ of color development solution was added to each section, and the section was observed under a microscope (Olympus Corporation). After color development, the section was rinsed by purified water to terminate the color development. Finally, re-dyeing (30 sec each time) was carried out twice
Table I. General characteristics of the patients.

\begin{tabular}{lcc}
\hline Item & Case & Ratio $(\%)$ \\
\hline Sex & & \\
Male & 42 & 55.26 \\
Female & 34 & 44.74 \\
Age (years) & & \\
$\quad<60$ & 36 & 47.36 \\
$\geq 60$ & 40 & 52.64 \\
Degree of differentiation & & \\
Highly differentiated adenocarcinoma & 36 & 47.36 \\
Moderately differentiated adenocarcinoma & 27 & 35.52 \\
Poorly differentiated adenocarcinoma & 13 & 17.11 \\
Tumor size & & \\
$<3$ cm & 27 & 35.53 \\
$3-5$ cm & 30 & 39.47 \\
$>5$ cm & 19 & 25.00 \\
\end{tabular}

using hematoxylin, and the sections were sealed using neutral balsam.

XPF expression was detected by immunohistochemistry. Under high magnification (x400), we randomly selected 8 non-overlapping visions of high magnification of each section, and 100 cells were selected in each vision. According to the proportion of positive cells in each section, we performed evaluation via semi-quantitative scoring method using the following criteria: i) 0 point for section with the ratio of positive cells $<5 \%$; ii) 1 point for section with the ratio of positive cells between 5 and $25 \%$; iii) 2 points for section with the ratio of positive cells between 26 and 50\%; iv) 3 points for section with the ratio of positive cells between 51 and $75 \%$; v) 4 points for section with the ratio of positive cells $>75 \%$. Staining degree of cells was: i) 0 point for cells without any color; ii) 1 point for cells in fine granule shape and canary yellow; iii) 2 points for cells in coarse granule shape and brown yellow; iv) 3 points for cells in small mass shape and dark brown. The total points of the above two indexes were taken as the total score. In terms of expression evaluation, expressions with a total score $\leq 3$ points were considered low expression, total score between 4 and 5 points moderate expression, and total score $\geq 6$ was considered high expression. In the evaluation of the results, a total score $\leq 3$ points was considered a negative result, and a total score $>3$ points was a positive result.

Statistical analysis. Data analysis was performed using SPSS 19.0 software (Chicago, IL, USA). Correlation between the XPF expression and clinicopathological indexes of gastric cancer was verified via single-factor Chi-square test. The Cox proportional hazards regression model was used in the analysis of influencing factors of patient prognosis. The Kaplan-Meier was used to analyze the survival rates of XPF-positive and -negative patients. Inspection results were analyzed using the log-rank, Breslow and Tarone-Ware tests. $\mathrm{P}<0.05$ indicated that the difference had statistical significance. 
Table II. Comparison of XPF expression between the two groups.

\begin{tabular}{lcccc}
\hline & & \multicolumn{2}{c}{ XPF expression } & \\
\cline { 3 - 4 } Group & $\mathrm{N}$ & $\begin{array}{c}\text { Negative } \\
\text { (case) }\end{array}$ & $\begin{array}{c}\text { Positive } \\
\text { cases }\end{array}$ & $\begin{array}{c}\text { Rases } \\
\text { positive } \\
\text { cases }(\%)\end{array}$ \\
\hline Observation group & 76 & 22 & 54 & 71.05 \\
Control group & 76 & 48 & 28 & 36.84 \\
$\chi^{2}$ & & & 16.551 & \\
P-value & & & $<0.0001$ & \\
\hline
\end{tabular}

XPF, xeroderma pigmentosum complementation group F.

\section{Results}

In comparison of XPF expression, we found that the positive expression rate $(71.05 \%)$ in the observation group was obviously higher than that $(36.84 \%)$ in the control group, and the difference in the intergroup comparison had statistical significance $(\mathrm{P}<0.05)$ (Table II and Fig. 1$)$.

XPF expression was significantly correlated with the family history and Laurén classification and the difference had statistical significance $(\mathrm{P}<0.05)$; XPF expression was not correlated with patient's age, sex, tumor site, quantity of mass, diameter of tumor, smoking, quantity of metastatic lymph nodes, infiltration depth and tumor node metastasis (TNM) staging, and the differences were not statistically significant (P>0.05) (Table III).

Factors affecting patient prognosis were analyzed using the Cox hazards model. The results suggested that XPF expression and TNM staging were independent factors affecting the prognosis of gastric cancer $(\mathrm{P}<0.05)$ (Table IV).

Kaplan-Meier survival analysis showed that the survival time of XPF-positive patients was shorter than that of XPF-negative patients. Inspection results using 3 different statistics were basically the same, and the differences were statistically significant $(\mathrm{P}<0.05)$ (Fig. 2 and Table V).

\section{Discussion}

In human cells, DNA in normal metabolism can be damaged due to the influences of various external factors, and such damage can often be accumulated, finally inducing a variety of cancers (8). In the human body, there are several different pathways for genetic repair, among which NER plays a major role. XPF gene, i.e., xeroderma pigmentosum complementation group, can encode XPF protein, a rate-limiting molecule in NER pathway; XPF protein, with the key effect in NER repair, can identify damaged $5^{\prime}$ end in DNA repair $(9,10)$. A variety of studies (11) have shown that XPF gene is correlated with the occurrence of various cancers, such as colon cancer, lung cancer, breast cancer and bladder cancer.

Significance of XPF expression in gastric cancer. Genetic damage will cause cell apoptosis, and sometimes, a minor damage can even induce cell apoptosis (12). However, the encoded product of XPF gene, a key enzyme in NER

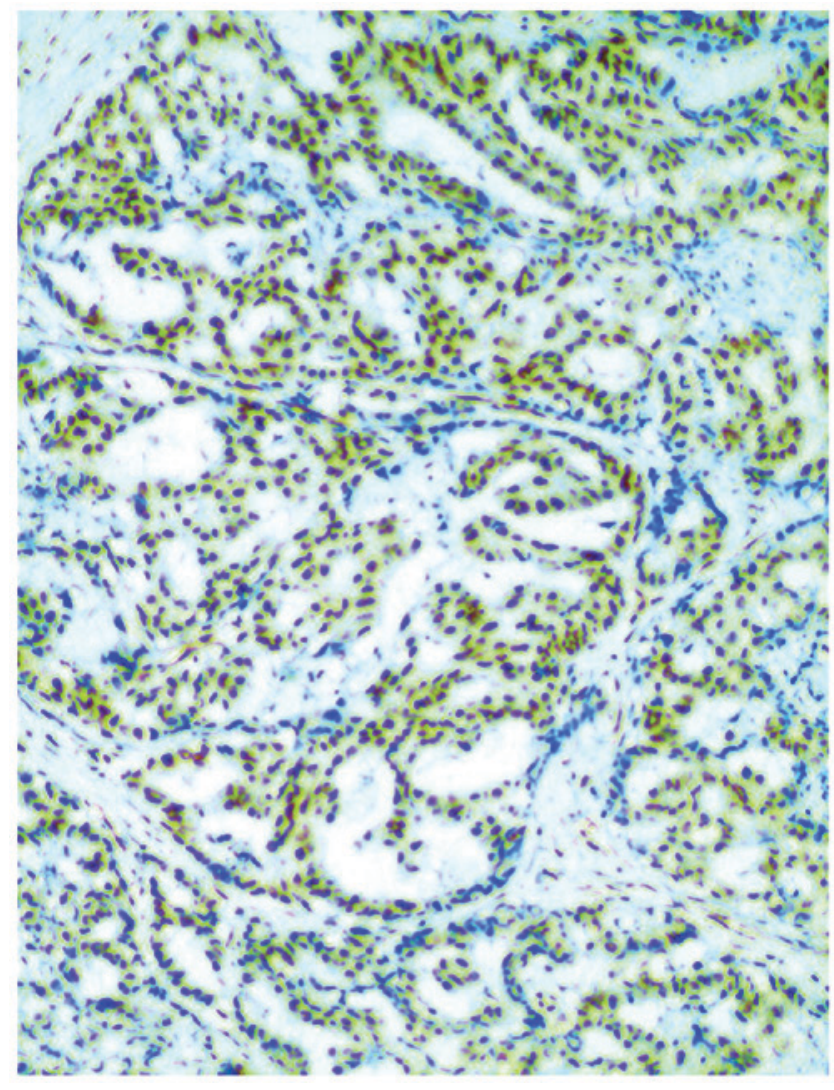

Figure 1. Xeroderma pigmentosum complementation group F (XPF)-positive cells in the observation group.

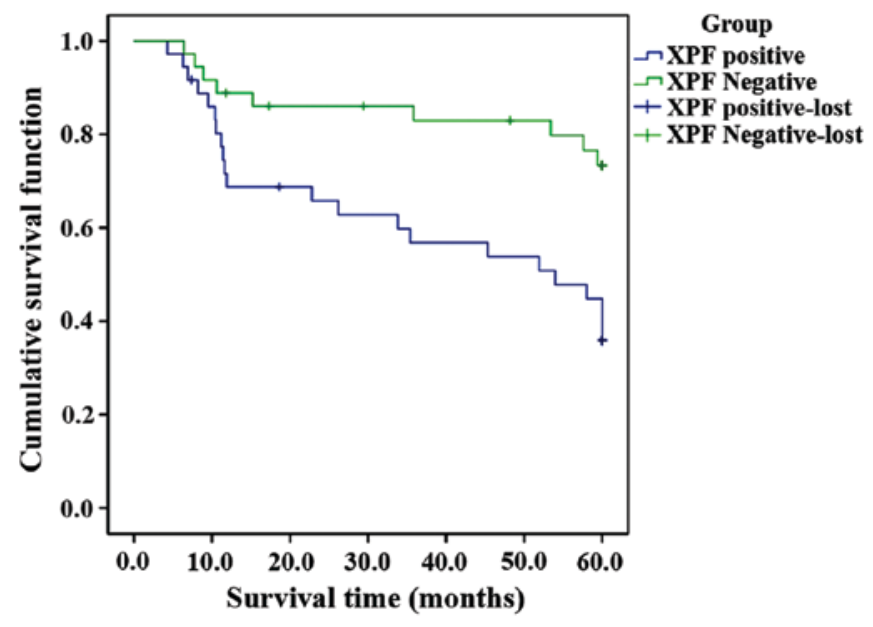

Figure 2. Kaplan-Meier survival analysis.

pathway, plays a critical role in anticancer activity, and, with the enhanced functions, can not only induce the antagonistic effect of gastric tumor cells on the platinum-based chemotherapy drugs, resulting in drug resistance and failure in chemotherapy, but also give rise to the abnormality in the repair of damaged DNA, leading to a significantly increased risk of carcinoma $(13,14)$. The activity of XPF gene is relatively high when the capability of cell repair is in a relatively high level; due to the stimulation generated by damage, NER will be activated to induce the XPF expression and increase the activity (15). According to the relevant study (16), XPF 
Table III. Correlation between XPF expression and each clinicopathological index.

\begin{tabular}{|c|c|c|c|c|c|c|}
\hline \multirow[b]{2}{*}{ Clinicopathological indexes } & \multirow[b]{2}{*}{ Cases } & \multicolumn{2}{|c|}{ XPF expression } & \multirow[b]{2}{*}{ Positive rate $(\%)$} & \multirow[b]{2}{*}{$\chi^{2}$} & \multirow[b]{2}{*}{ P-value } \\
\hline & & Negative case & Positive case & & & \\
\hline \multicolumn{7}{|l|}{ Sex } \\
\hline Male & 42 & 12 & 30 & 71.42 & 0.002 & 0.956 \\
\hline Female & 34 & 9 & 25 & 73.35 & & \\
\hline \multicolumn{7}{|l|}{ Age (year) } \\
\hline$<60$ & 36 & 11 & 25 & 69.44 & 0.001 & 0.968 \\
\hline$\geq 60$ & 40 & 11 & 29 & 72.50 & & \\
\hline \multicolumn{7}{|c|}{$\begin{array}{l}\text { History of liver cancer in immediate } \\
\text { family members }\end{array}$} \\
\hline Yes & 32 & 7 & 25 & 78.12 & 9.949 & 0.014 \\
\hline No & 44 & 23 & 21 & 52.27 & & \\
\hline \multicolumn{7}{|l|}{ Tumor site } \\
\hline Gastric body & 25 & 7 & 18 & 72.00 & 0.094 & 0.954 \\
\hline Gastric antrum & 36 & 11 & 25 & 69.44 & & \\
\hline Gastric cardia + gastric fundus & 15 & 4 & 11 & 73.33 & & \\
\hline \multicolumn{7}{|l|}{ Quantity of mass } \\
\hline 1 & 35 & 10 & 25 & 71.42 & 0.007 & 0.929 \\
\hline$\geq 2$ & 41 & 11 & 30 & 73.17 & & \\
\hline \multicolumn{7}{|l|}{ Diameter of tumor $(\mathrm{cm})$} \\
\hline$<5$ & 51 & 15 & 36 & 70.58 & 0.049 & 0.823 \\
\hline$\geq 5$ & 25 & 6 & 19 & 76.00 & & \\
\hline \multicolumn{7}{|l|}{ Laurén classification } \\
\hline Diffuse-type & 31 & 18 & 13 & 41.93 & 12.420 & 0.002 \\
\hline Mixed-type & 17 & 2 & 15 & 88.23 & & \\
\hline Intestinal-type & 28 & 7 & 21 & 75.00 & & \\
\hline \multicolumn{7}{|l|}{ Smoking } \\
\hline Yes & 31 & 7 & 24 & 77.42 & 0.309 & 0.578 \\
\hline No & 45 & 14 & 31 & 68.89 & & \\
\hline \multicolumn{7}{|c|}{ Lymphatic metastasis (lymph nodes) } \\
\hline$\leq 5$ & 46 & 13 & 33 & 71.74 & 0.012 & 0.912 \\
\hline$>5$ & 30 & 8 & 22 & 73.33 & & \\
\hline \multicolumn{7}{|l|}{ Infiltration depth } \\
\hline Mucosa and submucosa & 17 & 5 & 12 & 70.58 & 0.404 & 0.817 \\
\hline Muscular layer & 35 & 9 & 26 & 74.28 & & \\
\hline Serosal or subserosal & 24 & 8 & 16 & 66.67 & & \\
\hline \multicolumn{7}{|l|}{ TNM staging } \\
\hline I & 21 & 6 & 15 & 71.43 & 0.167 & 0.982 \\
\hline II & 24 & 6 & 18 & 75.00 & & \\
\hline III & 18 & 5 & 13 & 72.22 & & \\
\hline IV & 13 & 3 & 10 & 76.92 & & \\
\hline
\end{tabular}

$\mathrm{XPF}$, xeroderma pigmentosum complementation group $\mathrm{F}$.

expression in tumor tissues is obviously higher than that in the tissues adjacent to the tumors. In this study, we analyzed XPF expression in the gastric cancer tissues of 76 gastric cancer patients, and found that XPF expressions in the gastric cancer tissues were significantly higher than those in the tissues adjacent to the tumors $(\mathrm{P}<0.05)$.
Correlation betweenXPF expressionand clinicopathological indexes of gastric cancer. The results of this study showed that XPF expression was correlated with Laurén classification and family history; higher positive expression of XPF gene were found in the patients with immediate family history of liver cancer, and in mixed- or intestinal-type of Laurén 
Table IV. Analysis of factors affecting the prognosis of gastric cancer via the Cox hazards model.

\begin{tabular}{lccccr}
\hline Related factors & B & SE & Wald & OR (95\% CI) & P-value \\
\hline Sex & 0.042 & 0.013 & 0.543 & $0.567(0.726-0.936)$ & 0.436 \\
Age (years) & 0.028 & 0.009 & 0.332 & $0.964(0.824-1.137)$ & 0.202 \\
Smoking & -0.038 & 0.037 & 0.237 & $1.035(0.228-1.425)$ & 0.113 \\
Tumor site & -0.043 & 0.084 & 1.143 & $0.937(0.425-1.948)$ & 0.223 \\
Tumor size & 0.036 & 0.032 & 0.217 & $1.046(1.035-1.876)$ & 0.535 \\
Laurén classification & 0.015 & 0.042 & 1.085 & $0.875(0.532-1.452)$ & 0.247 \\
TNM staging & 0.485 & 0.052 & 5.012 & $2.025(1.023-3.627)$ & 0.003 \\
XPF expressions & 0.463 & 0.026 & 6.015 & $3.564(1.143-5.835)$ & 0.017 \\
Infiltration depth & 0.516 & 0.037 & 0.437 & $0.875(0.532-0.952)$ & 0.124 \\
Lymphatic metastasis & -0.028 & 0.141 & 0.767 & $1.025(0.623-1.627)$ & 0.215 \\
\hline
\end{tabular}

$\mathrm{XPF}$, xeroderma pigmentosum complementation group F; TNM, tumor node metastasis.

Table V. Comparison of inspection results using 3 different statistics for survival analysis.

\begin{tabular}{lccc}
\hline Type & $\chi^{2}$ value & df & P-value \\
\hline Log Rank (Mantel-Cox) & 9.205 & 1 & 0.002 \\
Breslow (Generalized Wilcoxon) & 8.196 & 1 & 0.004 \\
Tarone-Ware & 8.772 & 1 & 0.003 \\
\hline
\end{tabular}

classification, and the difference had statistical significance $(\mathrm{P}<0.05)$; XPF expression was not correlated with patient's sex, age, smoking habit, tumor site, diameter and quantity of tumor, infiltration depth, quantity of lymphatic metastasis and TNM-staging ( $P>0.05)$. Due to the genetic susceptibility of gastric cancer, many gastric cancer patients have a family history of gastric cancer; a relevant study (17) showed that incidence rate of gastric cancer of members with the family history of gastric cancer is 1.5-3 times higher than that of members without family history of gastric cancer. In history, the family of Napoleon was a typical example illustrating the genetic susceptibility in the family with the history of gastric cancer. Among those patients with the family history of gastric cancer, gene defect or mutation may be the major cause for canceration, and the risk of gastric cancer will be increased due to the inheritance of susceptibility gene (18). In Laurén classifications, non-tumor mucosal atrophy has been scarcely found in peripheral tissues of diffuse-type gastric cancer, but intestinal metaplasia and extensive atrophy are often associated with the intestinaland mixed-type gastric cancer. Thus, damage to gene in varying degrees may induce the translation of XPF proteins in different activity, which will lead to the differences in rates of positive expression (19).

Association between XPF expression and prognosis of gastric cancer. Currently, exact pathogenesis of gastric cancer has not been fully clarified; but many studies have shown that repair gene is not only correlated with the occurrence and development of gastric cancer, but also closely associated with the prognosis (20). In this study, we performed survival analysis for XPF-positive and -negative patients with gastric cancer, and the results showed that the survival time of XPF-negative patients was longer than that of XPF-positive patients $(\mathrm{P}<0.05)$; we selected clinicopathological indexes according to the prognostic factors of gastric cancer patients, performed regression analysis using Cox's hazards model, and results suggested that XPF was an independent prognostic factor. This suggested that positive expression of XPF gene, in addition to its critical effect on the formation of gastric cancer, exerts a larger effect on the distant metastasis or recurrence of tumor in the progressive stage. Thus, XPF can serve as an important index affecting the prognosis of gastric cancer patients (21).

In conclusion, increased XPF expression plays an important role in the occurrence, development and prognosis of gastric cancer, and can serve as an index for evaluating the prognosis of gastric cancer. This study was limited by sample size, and in-depth investigation into the XPF expression in tissues of human are expected.

\section{Acknowledgements}

Not applicable.

\section{Funding}

No funding was received.

\section{Availability of data and materials}

The datasets used and/or analyzed during the current study are available from the corresponding author on reasonable request.

\section{Authors' contributions}

PL analyzed the general information of the patients. PL and YM performed immunohistochemistry. Both authors read and approved the final manuscript. 


\section{Ethics approval and consent to participate}

Patients signed the informed consent. The study was approved by the Ethics Committee of the Second People's Hospital of Dezhou City (Dezhou, China).

\section{Patient consent for publication}

Not applicable.

\section{Competing interests}

The authors declare that they have no competing interests.

\section{References}

1. Ryu MH and Kang YK: ML17032 trial: Capecitabine/cisplatin versus 5-fluorouracil/cisplatin as first-line therapy in advanced gastric cancer. Expert Rev Anticancer Ther 9: 1745-1751, 2009.

2. Schinzari G, Cassano A, Orlandi A, Basso M and Barone C: Targeted therapy in advanced gastric carcinoma: The future is beginning. Curr Med Chem 21: 1026-1038, 2014.

3. Watari J, Chen N, Amenta PS, Fukui H, Oshima T, Tomita T, Miwa H, Lim KJ and Das KM: Helicobacter pylori associated chronic gastritis, clinical syndromes, precancerous lesions, and pathogenesis of gastric cancer development. World J Gastroenterol 20: 5461-5473, 2014

4. Olivero M, Dettori D, Arena S, Zecchin D, Lantelme E and Di Renzo MF: The stress phenotype makes cancer cells addicted to CDT2, a substrate receptor of the CRL4 ubiquitin ligase. Oncotarget 5: 5992-6002, 2014.

5. Marteijn JA, Lans H, Vermeulen W and Hoeijmakers JH: Understanding nucleotide excision repair and its roles in cancer and ageing. Nat Rev Mol Cell Biol 15: 465-481, 2014.

6. Vaezi A, Wang X, Buch S, Gooding W, Wang L, Seethala RR, Weaver DT, D'Andrea AD, Argiris A, Romkes M, et al: XPF expression correlates with clinical outcome in squamous cell carcinoma of the head and neck. Clin Cancer Res 17: 5513-5522, 2011.

7. Gregg SQ, Robinson AR and Niedernhofer LJ: Physiological consequences of defects in ERCC1-XPF DNA repair endonuclease. DNA Repair (Amst) 10: 781-791, 2011.

8. Liu L, Chang Y, Ye J and Kumar S: Abstract 1923: Distinct evolutionary and mutational patterns in oncogenes and tumor suppressor genes. Cancer Res 75: abs. 1923, 2015. doi: 10.1158/1538-7445.AM2015-1923.

9. Liu J, He C, Xing C and Yuan Y: Nucleotide excision repair related gene polymorphisms and genetic susceptibility, chemotherapeutic sensitivity and prognosis of gastric cancer. Mutat Res 765: 11-21, 2014.
10. Ying M, Deng XD, Yu F, Zhang W, Wang SX, Liu Y and Liu H: Association of XPF, levels and genetic polymorphism with susceptibility to ischemic stroke. J Mol Neurosci 59: 1-9, 2016.

11. Cheng HB, Xie C, Zhang RY, Hu SS, Wang Z and Yue W: Xeroderma pigmentosum complementation group $\mathrm{F}$ polymorphisms influence risk of glioma. Asian Pac J Cancer Prev 14: 4083-4087, 2013.

12. Singh VV, Dutta D, Ansari MA, Dutta S, Chandran B and Longnecker R: Kaposi's sarcoma-associated herpesvirus induces the ATM and H2AX DNA damage response early during de novo infection of primary endothelial cells, which play roles in latency establishment. J Virol 88: 2821-2834, 2014.

13. Wei ZH, Guo WH, Wu J, Suo WH and Fu GH: A nonsense mutation in the Xeroderma pigmentosum complementation group $\mathrm{F}$ (XPF) gene is associated with gastric carcinogenesis. Gene 537: 238-244, 2014.

14. Graf N, Ang WH, Zhu G, Myint M and Lippard SJ: Role of endonucleases XPF and XPG in nucleotide excision repair of platinated DNA and cisplatin/oxaliplatin cytotoxicity. Chembiochem 12: 1115-1123, 2011.

15. Matsumoto S, Fischer ES, Yasuda T, Dohmae N, Iwai S, Mori T, Nishi R, Yoshino K, Sakai W, Hanaoka F, et al: Functional regulation of the DNA damage-recognition factor DDB2 by ubiquitination and interaction with xeroderma pigmentosum group C protein. Nucleic Acids Res 43: 1700-1713, 2015.

16. Facista A, Nguyen H, Lewis C, Prasad AR, Ramsey L, Zaitlin B, Nfonsam V, Krouse RS, Bernstein H, Payne CM, et al: Deficient expression of DNA repair enzymes in early progression to sporadic colon cancer. Genome Integr 3: 3, 2012.

17. Jiang X, Tseng CC, Bernstein L and Wu AH: Family history of cancer and gastroesophageal disorders and risk of esophageal and gastric adenocarcinomas: A case-control study. BMC Cancer 14: 60, 2014

18. Reddy KM, Chang JI, Shi JM and Wu BU: Risk of gastric cancer among patients with intestinal metaplasia of the stomach in a United States Integrated Healthcare System. Clin Gastroenterol Hepatol 14: 1420-1425, 2016.

19. Caruso RA, Branca G, Fedele F, Parisi A, Finocchiaro G, Ieni A and Rigoli L: Eosinophil-specific granules in tumor cell cytoplasm: Unusual ultrastructural findings in a case of diffusetype gastric carcinoma. Ultrastruct Pathol 39: 226-230, 2015.

20. Khaleghian M, Shakoori A, Razavi AE and Azimi C: Relationship of amplification and expression of the C-MYC gene with survival among gastric cancer patients. Asian Pac J Cancer Prev 16: 7061-7069, 2015

21. Li J, Zuo X, Lv X, Kong F, Xu W and Yang S: Association of DNA repair gene polymorphisms with response to chemotherapy and prognosis of gastric cancer in a Chinese population. Tumour Biol 35: 7569-7574, 2014.

This work is licensed under a Creative Commons Attribution-NonCommercial-NoDerivatives 4.0 International (CC BY-NC-ND 4.0) License. 